\title{
Multidrug-resistant Aeromonas hydrophila causing fatal bilateral necrotizing fasciitis in an immunocompromised patient: a case report
}

\author{
Alejandra Ugarte-Torres ${ }^{1 \dagger}$, Sarah Perry ${ }^{1 \dagger}$, Angela Franko ${ }^{2}$ and Deirdre L Church ${ }^{1,2,3^{*}}$
}

\begin{abstract}
Background: Aeromonas hydrophila is a water-dwelling, gram-negative rod-shaped bacterium, associated with diarrheal illness and, less commonly, necrotizing skin and soft tissue infections, especially among immunocompromised patients. Necrotizing fasciitis is associated with a high mortality rate, especially when caused by Aeromonas spp. Our patient was infected with an extremely aggressive form of multidrug-resistant Aeromonas spp. that produced both an extended-spectrum $\beta$-lactamase and an AmpC enzyme. Aeromonads are being recognized as important emerging pathogens because of their inherent antibiotic resistance profiles compounded by other virulence factors. These difficult-to-treat organisms can have significant implications in both clinical and public health settings.

Case presentation: A 37-year-old Caucasian male with immunosuppression due to aplastic anemia being treated with cyclosporine, presented to hospital with relapsed disease. While in hospital, he subsequently developed overwhelming sepsis secondary to bilateral lower extremity necrotizing fasciitis. The necrotizing fasciitis was caused by a multidrug-resistant strain of A. hydrophila. Despite broad-spectrum antibiotics and aggressive surgical debridement, he succumbed to this severe invasive infection.

Conclusions: Necrotizing fasciitis caused by Aeromonas spp. is a rare infection that may have a poor clinical outcome, particularly if the diagnosis is delayed and/or the organism is highly virulent and multidrug resistant. Enhanced education of clinicians and microbiologists is required to prevent unnecessary complications and improve survival.
\end{abstract}

Keywords: Necrotizing fasciitis, Multidrug resistance, Aeromonas hydrophila

\section{Background}

Aeromonas species are gram-negative, non-sporulating, facultative, anaerobic small bacilli with a ubiquitous distribution [1]. They are water-dwelling, opportunistic pathogens commonly found not only in environmental sources such as fresh and brackish water, seafood, meat, and vegetables, but have also been isolated from chlorinated water, including hospital water supplies [2]. Aeromonas spp. infection has a variety of clinical

\footnotetext{
* Correspondence: deirdre.church@cls.ab.ca

${ }^{\dagger}$ Alejandra Ugarte Torres and Sarah Perry contributed equally to this work.

'Department of Medicine, University of Calgary, 9-3535 Research Rd NW,

Calgary, AB T2L 2K8, Canada

${ }^{2}$ Department of Pathology and Laboratory Medicine, University of Calgary,

Calgary, Canada

Full list of author information is available at the end of the article
}

presentations, including gastroenteritis, hepatobiliary tract infection, pneumonia, skin and soft tissue infections, empyema, meningitis, septic arthritis, osteomyelitis, endocarditis, and bacteremia [3]. Currently, there are more than 20 species identified, but only 7 have been recognized as human pathogens, namely $A$. hydrophila, A. caviae, A. veronii biovar sobria, $A$. veronii biovar veronii, A. jandaei, A. trota, and A. schubertii, with the first three being the most common [4]. Invasive Aeromonas infections usually occur amongst immunocompromised individuals, primarily in patients with solid or hematologic malignancies or hepatobiliary disease, but healthy individuals may also be affected after sustaining traumatic and crush injuries, near drowning events, and burns [2].

(c) The Author(s). 2018 Open Access This article is distributed under the terms of the Creative Commons Attribution 4.0 International License (http://creativecommons.org/licenses/by/4.0/), which permits unrestricted use, distribution, and reproduction in any medium, provided you give appropriate credit to the original author(s) and the source, provide a link to the Creative Commons license, and indicate if changes were made. The Creative Commons Public Domain Dedication waiver (http://creativecommons.org/publicdomain/zero/1.0/) applies to the data made available in this article, unless otherwise stated. 
Necrotizing fasciitis (NF) has a rapid clinical progression that results in septic shock and associated multiorgan failure. Severe necrosis of the skin and underlying soft tissues is mediated by the release of bacterial toxins and proteases that lead to extensive inflammation $[5,6]$. Some of the recognized risk factors associated with NF are immunosuppression, diabetes mellitus, alcoholism, end-stage renal disease, malignancy, chemotherapy, and previous surgery or trauma [7]. Polymicrobial NF, or type $\mathrm{I}$, is the most common presentation (approximately $80 \%$ of cases), followed by monomicrobial, or type II (15\% of cases), classically caused by group A Streptococcus [8]. Less than $5 \%$ of NF cases are caused by water-born organisms, most commonly Vibrio vulnificus and Aeromonas spp. Although the incidence of NF is low, estimated at 0.04 cases per 1000 persons-years in the United States [9], both types of NF are associated with a high mortality, ranging from 17 to $34 \%[1,2]$, and cases caused by Aeromonas spp. have the highest reported mortality rate of up to $60 \%$ [10].

Herein, we report a case of a young immunocompromised male undergoing treatment for relapsed aplastic anemia that developed fatal sepsis secondary to bilateral leg NF caused by an A. hydrophila strain that was multidrug resistant.

\section{Case presentation}

A 37-year-old Caucasian male with a known history of aplastic anemia (AA), presented to a rural hospital after a ground level fall. AA was diagnosed 10 months earlier after he was investigated for pancytopenia. A bone marrow biopsy showed cellularity of only $10 \%$ and the presence of a small paroxysmal nocturnal hemoglobinuria clone (less than $0.2 \%$ ). He received standard combination treatment for AA with cyclosporine $225 \mathrm{mg}$ orally twice daily, horse anti-thymocyte globulin (ATG) $40 \mathrm{mg} / \mathrm{kg}$ daily for 4 consecutive days, and prednisone $1 \mathrm{mg} / \mathrm{kg}$ daily. His other medications included daily Pantoloc $40 \mathrm{mg}$ orally, daily Valtrex $500 \mathrm{mg}$ orally, and daily Dapsone $50 \mathrm{mg}$ orally for Pneumocystis jirovecii prophylaxis due to a reported allergy to trimethoprim/sulfamethoxazole. He had recently quit smoking and denied alcohol use but actively used other recreational drugs, including marijuana, cocaine, and methamphetamine. He was unemployed. He had no known other medical co-morbidities and was taking no other medications prior to developing AA. The etiology of AA was felt to be idiopathic because he had no improvement after an initial trial of sobriety. AA improved following immunosuppressive therapy and, although human leukocyte antigen typing was performed, a subsequent bone marrow transplant was deferred not only because of the medical therapeutic response but also due to his ongoing recreational drug use. Although he was no longer transfusion dependent a month after starting immunosuppressive therapy, his treatment compliance waned overtime due to regular ongoing recreational drug use of cocaine and methamphetamines. He routinely used unsterilized tap water for illicit drug injections, but he denied other exposure to fresh or salt water sources at home or in the community.

On presentation to the emergency department he was not in distress, with a heart rate of $90 \mathrm{bpm}$ and a blood pressure of $116 / 59$. Severe pallor was noted upon examination, as well as a petechial rash and mild ecchymoses (Fig. 1). The rest of his physical assessment was normal, including a neurological examination. Admission bloodwork revealed severe pancytopenia with hemoglobin of $22 \mathrm{~g} / \mathrm{L}$, a platelet count of $1 \times 10^{9} / \mathrm{L}$, a white blood cell count of $3.7 \times 10^{9} / \mathrm{L}$, and an absolute neutrophil count of $0.2 \times 10^{9} / \mathrm{L}$ (reticulocytes were not sent at admission, but 2 weeks into his hospitalization his absolute reticulocyte count was $12 \times 10^{9} / \mathrm{L}$ with a reticulocyte percentage of 0.5 ). All other admission blood work was normal, including liver function tests (total bilirubin $9 \mu \mathrm{mol} / \mathrm{L}$ (reference $<21 \mu \mathrm{mol} / \mathrm{L}$ ), alanine aminotransferase $13 \mu \mathrm{mol} /$ $\mathrm{L}$ (reference $<41 \mu \mathrm{mol} / \mathrm{L}$ ), alkaline phosphatase $66 \mathrm{U} / \mathrm{L}$ (reference 30-130 U/L)) and renal function tests (creatinine $63 \mu \mathrm{mol} / \mathrm{L}$ (reference 59-104 $\mu \mathrm{mol} / \mathrm{L}$ ), glomerular filtration rate $120 \mathrm{~mL} / \mathrm{min}$ (reference $<59 \mathrm{~mL} / \mathrm{min}$ )). He was stabilized and transferred to a tertiary care center where he was restarted on treatment for relapsed AA with a regimen that included cyclosporine $(5 \mathrm{mg} / \mathrm{kg} /$ day) and prednisone $30 \mathrm{mg}$ daily in addition to five doses of ATG. He remained transfusion dependent throughout his hospitalization.

On day 10 after admission, he developed generalized, mild (3/10), colicky abdominal pain with an associated fever $>38.5{ }^{\circ} \mathrm{C}$. He was started empirically on piperacillin-tazobactam (PTZ) 3.375 gm intravenously every 6 hours. Two sets of blood cultures, each consisting of an anaerobic and aerobic BacT/Alert bottle (bioMérieux, Laval, Quebec), were collected peripherally and from his central line. E. coli grew in each bottle set at 10 and 11 hours, respectively. He then developed watery, non-bloody bowel movements, 3-4 times a day, associated with rectal pain. Real-time PCR for Clostridium difficile A/B toxin on a stool sample was negative. Computerized tomography of the abdomen and pelvis was also unremarkable. Repeat blood cultures were negative at 24 and 48 hours after the initial positive set. He improved dramatically after 7 days of intravenous PTZ and was stepped down to oral ciprofloxacin $500 \mathrm{mg}$ orally twice daily to complete a further 7 days of therapy.

On day 19 of admission he developed acute continuous severe (9/10), non-radiating dull rectal pain, associated with a high-grade fever $\left(40.4{ }^{\circ} \mathrm{C}\right)$. Vancomycin $1.5 \mathrm{~g}$ intravenously every 12 hours and metronidazole $500 \mathrm{mg}$ orally twice daily were empirically started and ciprofloxacin was 
continued in the same dosage. Blood cultures that were collected from peripheral venipuncture and a peripherally inserted central catheter line grew $A$. hydrophila at 11 hours. The peripherally inserted central catheter line was immediately removed the next day (day 20 after admission). The same day he also began to complain of vague, mild, bilateral leg pain. Delayed serum sickness due to recent ATG administration was considered a possible cause for his new symptoms because clinical examination did not show erythema, edema, or deformities on either of his legs. However, sustained bacteremia was diagnosed by recovery of $A$. hydrophila from repeat blood cultures (i.e., one anaerobic and aerobic bottle set from two peripheral venipunctures) positive after 11 and 16 hours of incubation. Bilateral leg pain steadily worsened in intensity (10/ 10) over the next 48 hours, and the area of distribution of pain extended to the lateral aspect of the right thigh although physical examination remained unremarkable. Creatinine kinase was increased at $470 \mathrm{U} / \mathrm{L}$ (normal range for males, 0-195 U/L). Ultrasound venous Doppler of both legs also showed no evidence of deep venous thrombosis. However, magnetic resonance images of both legs showed extensive bilateral patchy multi-compartment muscular and fascial inflammatory changes highly concerning for NF (Fig. 2a, b).

Urgent initial surgical debridement was performed that evening. An extensive four-compartment fasciotomy, debridement, and myomectomy were performed on both legs. Extensive 'dishwater' purulent material was found in multiple compartments of both legs, including (1) the superficial posterior compartment between the gastrocnemius and soleus muscles, and (2) the lateral deep compartment. There was also clinical evidence of severe muscle necrosis of the tibialis anterior muscles in the anterior compartment of both legs. He was admitted to the Intensive Care Unit post-operatively. After consultation with the Infectious Diseases service and review of the antibiotic susceptibility profile of the previously isolated $A$. hydrophila strain, antibiotics were changed to meropenem $1000 \mathrm{mg}$ intravenously every 8 hours and clindamycin $600 \mathrm{mg}$ intravenously every 8 hours. High dose intravenous immunoglobulin $(2 \mathrm{~g} / \mathrm{kg})$ was also given. All prior antibiotics were discontinued.

Gram stain of tissue samples from the right tibialis anterior muscle showed no neutrophils but that gramnegative bacilli were present, and subsequently grew a

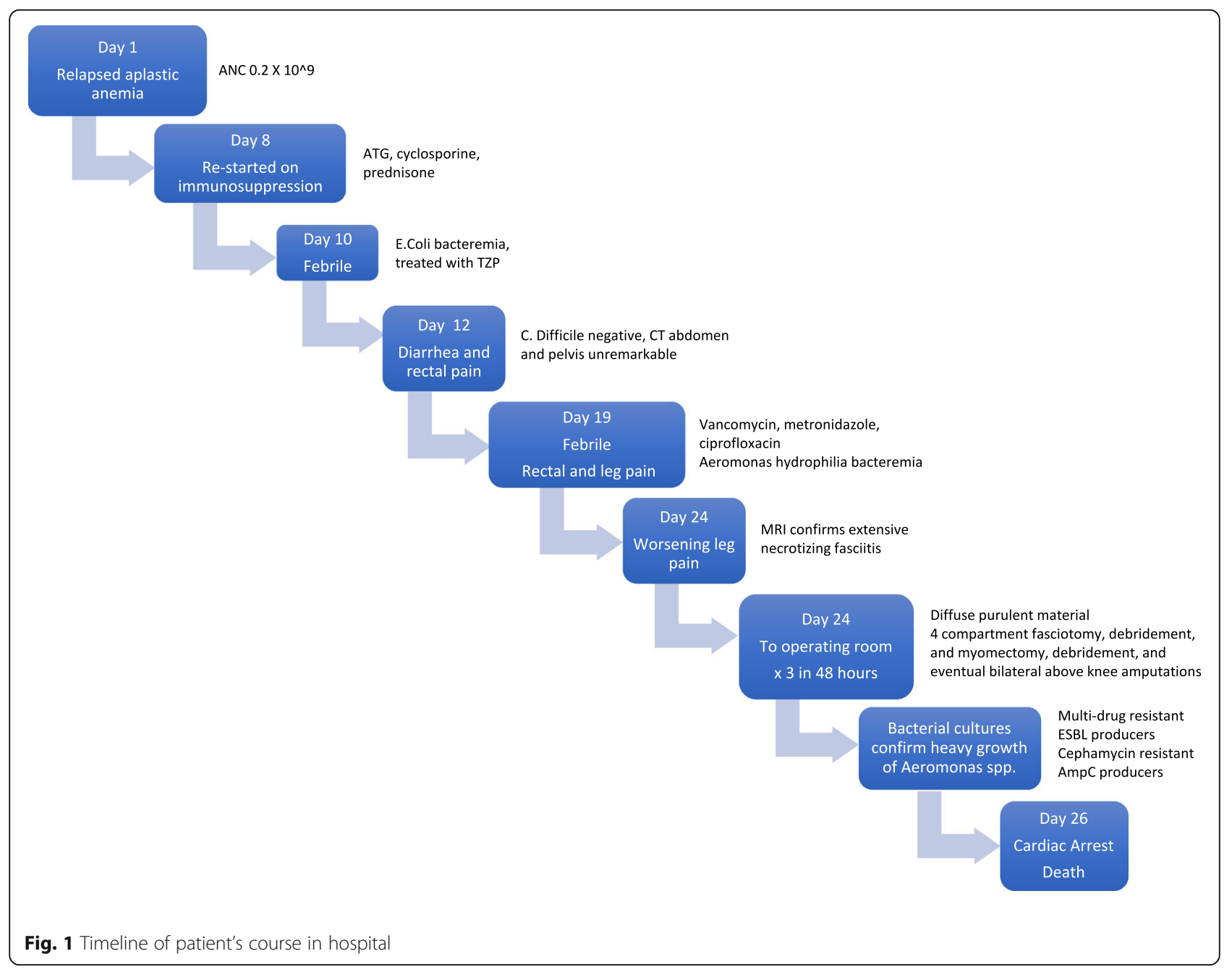



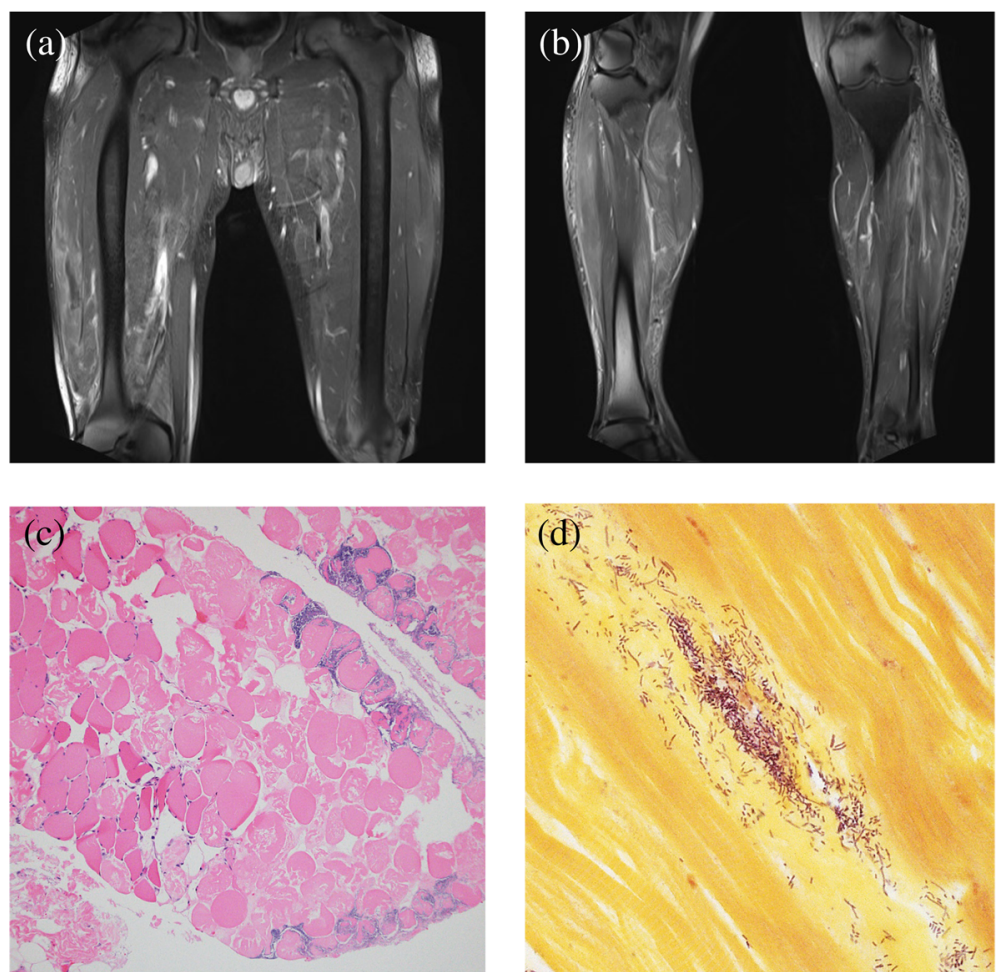

Fig. 2 Coronal views of enhanced bilateral magnetic resonance imaging T2 image of hips (a) and legs (b). Muscular edema of vastus intermedius, vastus lateralis, mid rectus femoris, abductor magnus, abductor brevis, gracilis, and gastrocnemius associated with extensive multi-compartmental fascial edema bilaterally. No rim-enhancing collections or gas were noted. c Histologic image of necrotic muscle with associated loss of nuclei and striations. There are dense aggregations of bacteria infiltrating the muscle with no associated inflammatory response. Inset shows normal viable muscle for comparison. $\mathbf{d}$ Gram stain with numerous gram-negative bacilli, consistent with Aeromonas hydrophila

heavy amount of $A$. hydrophila. Gram stain and anaerobic culture from the right vastis lateralis muscle also did not show the presence of neutrophils or organisms but grew scant amounts of $A$. hydrophila. A genus-level identification as Aeromonas was obtained for all isolates from blood and tissue samples by matrix-assisted laser desorption ionization-time of flight (MALDI-TOF) mass spectrometry using a VITEK MS (bioMérieux, Laval, Quebec, Canada); since this technique has an accuracy of identification rate of $80-90 \%$ for species-level identification of Aeromonas [11], all isolates were also analyzed using in-house bi-directional 16S rRNA gene cycle sequencing of the V1-V3 (approximately first $500 \mathrm{bp}$ ), as previously described [12]. Broth microdilution susceptibility panel testing was performed and interpreted using published guidelines [13]. All isolates were multidrug resistant to ampicillin, ceftriaxone, ciprofloxacin, and trimethoprim/sulfamethoxazole but susceptible to meropenem and tetracycline. The isolates were confirmed to produce an extended-spectrum $\beta$-lactamase (ESBL) using published guidelines and the Mast Disc Test (Mast Group Ltd., Merseyside, UK) [13]. Production of an
AmpC $\beta$-lactamase was shown by resistance to cefoxitin disk $(30 \mu \mathrm{g})$ testing and the Mast Disc test (Mast Group Ltd.).

Two additional extensive surgical procedures for removal of necrotic tissue from both legs were undertaken in the next 24 hours. Bilateral above-knee amputations were performed during the last debridement as a lifesaving measure because of extensive rapid progression of bilateral leg necrosis, and the patient's rapid clinical deterioration with severe unremittent hemodynamic instability during the operation. Post-operatively, he required aggressive resuscitation for septic shock in the Intensive Care Unit with intractable hyperkalemia and severe acidosis, and anuric acute kidney failure (creatinine $210 \mu \mathrm{mol} / \mathrm{L}$; normal range for males, $50-120 \mu \mathrm{mol} /$ L). Despite all therapeutic interventions, the patient went into cardiac arrest and passed away within 2 hours after the final surgery.

Post-mortem examination at autopsy revealed findings related to the underlying $\mathrm{AA}$, and evidence of septic shock secondary to extensive bilateral lower limb necrotizing myofasciitis. The bone marrow was markedly 
hypocellular and there was splenic enlargement at $331 \mathrm{~g}$. The heart was enlarged (536 g). Cardiomegaly was likely a compensatory response to the AA due to the absence of atherosclerotic and hypertensive cardiovascular disease. In keeping with the patient's severe septic shock, there was marked centrilobular necrosis of the liver, as well as petechial hemorrhages of the skin, heart, pleural surfaces, kidneys, and liver capsule. Histologic examination of skin and muscle from the left thigh showed necrosis of the muscle and deep subcutaneous adipose tissue, admixed with dense collections of gram-negative bacilli (Fig. 2c, d). However, in keeping with the AA, there was notably an absence of an acute inflammatory response.

\section{Discussion and conclusions}

In summary, this 37-year-old male with AA developed rapidly progressive bilateral NF from $A$. hydrophila that produced both an ESBL and $A m p C \beta$-lactamase, and was confirmed to be multidrug resistant to multiple classes of antibiotics. Similar cases caused by multidrug-resistant strains of $A$. hydrophila have not previously been reported. The rapid clinical course in this severely immunocompromised man is also attributable to delayed diagnosis. This unique case demonstrates that physicians must have a high level of suspicion for necrotizing skin and soft tissue infection (NSSTI) in patients with increasing pain and creatinine kinase levels despite an unremarkable clinical examination.

NSSTIs caused by Aeromonas spp. infection are rare but are associated with very high mortality, reportedly between 60 and $75 \%$ in immunocompromised hosts [4]. NSSTI caused by Aeromonas spp. is the second most common clinical presentation caused by these organisms after diarrheal illness. Invasive Aeromonas infections have been reported in patients with underlying hematological conditions, including those with leukemia, lymphoma, and hematopoietic stem cell transplant recipients $[14,15]$. Similar to our case, most of the previously reported NF cases have been associated with fatal septic shock and multi-organ failure $[14,16]$. There is no history of preceding trauma or water exposure in $20-30 \%$ of patients with Aeromonas-related sepsis or NSSTI.

Patients who develop NSSTI from Aeromonas spp. are often found to have gastrointestinal colonization with this organism [17]. The pathogenesis of the severe NSSTI in our case most likely occurred by a similar mechanism given the preceding gastrointestinal symptoms and associated E. coli bacteremia. Although he was asymptomatic on admission, severe bilateral leg pain acutely developed shortly after the onset of gastrointestinal symptoms. Most likely, secondary endogenous bacteremia occurred due to bacterial gut translocation and resulted in the subsequent seeding in the soft tissues and muscles of both legs. Unfortunately, there was no stool culture performed to prove Aeromonas colonization. Although intestinal colonization with Aeromonas spp. in otherwise healthy individuals has been reported to be very low $[18,19]$, we surmise that this was the probable source in our patient given his severe immunosuppression. Alternatively, A. hydrophila infection may have been caused by the use of contaminated tap water for injection of recreational drugs while in hospital - something suspected but never objectively proven by his medical team. This mechanism is considered less likely because there were no other nosocomial infections due to Aeromonas spp. reported by Infection Prevention and Control surveillance data throughout the period of his admission.

Aeromonas spp. are relatively common in some ecologic niches, particularly salt or fresh water environments [2]. Environmental studies have demonstrated that these organisms do not routinely contaminate drinking and surface water samples or food, which leads to the 'selection' of specific pathogenic strains of Aeromonas spp. to produce invasive infection [20]. Pathogenic Aeromonas spp. have also been shown to produce several virulence factors including aerolysin-related cytotoxic enterotoxin. This endotoxin causes diarrhea and severe tissue damage by inducing apoptosis of host cells [21]. Multiple other proteins have also been linked to tissue damage, such as VIP-2, which not only has cytotoxic effects [22] but also inhibits bacterial phagocytosis. Two other mechanisms are associated with the pathogenesis of Aeromonas infections, namely its capacity to form biofilms and the high hemolytic activity of aerolysin-related cytotoxic enterotoxin [23]. Aeromonas $s p p$. also produces several enzymes that have caseinase and elastase activity [24], which may also contribute to its ability to invade tissue and cause NF.

Finally, the multi-drug resistance profile of the A. hydrophila isolate in our patient was another major virulence factor that likely contributed to uncontrollable invasive infection and ultimate fatality in this case. On prior reports, drug resistance did not influence mortality [3], but none of the previously reported invasive Aeromonas spp. cases were caused by a highly multidrug-resistant strain as occurred herein. The major resistance mechanism reported to date for most Aeromonads is an inducible chromosomal $\beta$-lactamase $[25,26]$, including production of an ESBL and/or a metallo- $\beta$-lactamase active against carbapenems [27]. On a recent report from clinical isolates in France, only $2 \%$ were resistant to third-generation cephalosporins and $32 \%$ of isolates were resistant to carbapenems [28]. AmpC $\beta$-lactamase production by Aeromonas $s p p$. is uncommon but has primarily been reported in isolates from environmental samples and stool samples from 
adults and children in India [29]. The most common metallo- $\beta$-lactamase produced by Aeromonas spp. is the CphA type, other types detected are VIM and IMP encoded on an integron and a plasmid, respectively [25, 30]. Aeromonas species were thought to be universally susceptible to fluoroquinolones [1], but the strain recovered from our case demonstrated high-level resistance to fluoroquinolones.

NF caused by Aeromonas spp. is rare but may result in high morbidity and mortality if a diagnosis is delayed and the organism is highly virulent and multidrug resistant, as demonstrated by our case. Enhanced education of clinicians and microbiologists is required to improve patient outcomes. Clinicians need to be aware of this organism's potential to cause severe NSSTI, especially amongst immunocompromised patients or in normal hosts following traumatic injuries. Invasive NF infections due to Aeromonas spp. should be treated aggressively with surgical debridement and broad-spectrum antibiotics confirmed by laboratory testing to have documented susceptibility and clinical efficacy.

\section{Abbreviations}

AA: aplastic anemia; ATG: anti-thymocyte globulin; ESBL: extended spectrum $\beta$-lactamase; NF: necrotizing fasciitis; NSSTI: necrotizing skin and soft tissue infections

\section{Funding}

This case report was performed as a resident/fellow project. Publication of this manuscript was supported by the University of Calgary.

\section{Availability of data and materials}

The datasets used and/or analyzed during the current study are available from the corresponding author on reasonable request.

\section{Authors' contributions}

AUT and SP participated in acquisition of data and drafting of the manuscript. AF revised the manuscript critically for important intellectual content. DLC revised the manuscript critically for important intellectual content and supervised the writing of the manuscript. All authors read and approved the final manuscript.

\section{Ethics approval and consent to participate}

The patient is deceased. Written informed consent was obtained from the patient's next-of-kin for autopsy for the use of bodily tissues and organs for future medical education and research purposes (which is noted in the official autopsy report).

\section{Consent for publication}

Written informed consent was obtained from the patient's next-of-kin for publication of this case report and any accompanying images. A copy of the written consent is available for review by the Editor-in-Chief of this journal.

\section{Competing interests}

The authors declare that they have no competing interests.

\section{Publisher's Note}

Springer Nature remains neutral with regard to jurisdictional claims in published maps and institutional affiliations.

\section{Author details}

'Department of Medicine, University of Calgary, 9-3535 Research Rd NW, Calgary, AB T2L 2K8, Canada. ${ }^{2}$ Department of Pathology and Laboratory Medicine, University of Calgary, Calgary, Canada. ${ }^{3}$ Department of Medicine,
Snyder Institute for Chronic Diseases, Cummings School of Medicine, University of Calgary, Calgary, Canada.

Received: 13 January 2018 Accepted: 21 September 2018

Published online: 01 November 2018

\section{References}

1. Janda JM. Recent advances in the study of the taxonomy, pathogenicity, and infectious syndromes associated with the genus Aeromonas. Clin Microbiol Rev. 1991;4:397-410.

2. Janda JM, Abbott SL. The genus Aeromonas: taxonomy, pathogenicity, and infection. Clin Microbiol Rev. 2010;23:35-73.

3. Ko WC, Lee HC, Chuang YC, Liu CC, Wu JJ. Clinical features and therapeutic implications of 104 episodes of monomicrobial Aeromonas bacteraemia. J Inf Secur. 2000;40:267-73.

4. Janda JM, Guthertz LS, Kokka RP, Shimada T. Aeromonas species in septicemia: laboratory characteristics and clinical observations. Clin Infect Dis. 1994;19:77-83.

5. Minnaganti VR, Patel PJ, lancu D, Schoch PE, Cunha BA. Necrotizing fasciitis caused by Aeromonas hydrophila. Heart Lung. 2000;29:306-8.

6. Anaya DA, McMahon K, Nathens AB, Sullivan SR, Foy H, Bulger E. Predictors of mortality and limb loss in necrotizing soft tissue infections. Arch Surg. 2005;140:151-7. discussion 158

7. Chen IC, Li WC, Hong YC, Shie SS, Fann WC, Hsiao CT. The microbiological profile and presence of bloodstream infection influence mortality rates in necrotizing fasciitis. Crit Care. 2011:15:R152.

8. Giuliano A, Lewis F Jr, Hadley K, Blaisdell FW. Bacteriology of necrotizing fasciitis. Am J Surg. 1977;134:52-7.

9. Ellis Simonsen SM, van Orman ER, Hatch BE, Jones SS, Gren LH, Hegmann $K T$, Lyon JL. Cellulitis incidence in a defined population. Epidemiol Infect. 2006;134:293-9.

10. Spadaro S, Berselli A, Marangoni E, Romanello A, Colamussi MV, Ragazzi R, Zardi S, Volta CA. Aeromonas sobria necrotizing fasciitis and sepsis in an immunocompromised patient: a case report and review of the literature. J Med Case Rep. 2014;8:315.

11. Chen PL, Lee TF, Wu CJ, Teng SH, Teng LJ, Ko WC, Hsueh PR. Matrix-assisted laser desorption ionization-time of flight mass spectrometry can accurately differentiate Aeromonas dhakensis from A. hydrophila, A. caviae, and A. veronii. J Clin Microbiol. 2014:52:2625-8.

12. Miller RJ, Chow B, Pillai D, Church D. Development and evaluation of a novel fast broad-range 165 ribosomal DNA PCR and sequencing assay for diagnosis of bacterial infective endocarditis: multi-year experience in a large Canadian healthcare zone and a literature review. BMC Infect Dis. 2016;16:146.

13. Clinical Laboratory Standards Institute (CLSI). Performance Standards for Antimicrobial Susceptibility Testing. Approved Guideline M100-S27. Wayne, PA: CLSI: 2017.

14. Martino R, Santamaria A, Pericas R, Sureda A, Brunet S. Acute rhabdomyolysis and myonecrosis complicating aeromonas bacteremia in neutropenic patients with hematologic malignancies: report of two cases. Haematologica. 1997:82:692-4.

15. Tsai MS, Kuo CY, Wang MC, Wu HC, Chien CC, Liu JW. Clinical features and risk factors for mortality in Aeromonas bacteremic adults with hematologic malignancies. J Microbiol Immunol Infect. 2006;39:150-4.

16. Ko WC, Chuang YC. Aeromonas bacteremia: review of 59 episodes. Clin Infect Dis. 1995;20:1298-304.

17. Sherlock CH, Burdge DR, Smith JA. Does Aeromonas hydrophila preferentially colonize the bowels of patients with hematologic malignancies? Diagn Microbiol Infect Dis. 1987;7:63-8.

18. Figueras MJ. Clinical relevance of Aeromonas sM503. Rev Med Microbiol. 2005:16(4):145-53.

19. Altwegg M, Geiss HK, Freij BJ. Aeromonas as a human pathogen. CRC Crit Rev Microbiol. 2008;16:253-86.

20. Borrell N, Fiqueras MJ, Guarro J. Phenotypic identification of Aeromonas genomospecies from clinical and environmental sources. Can J Microbiol. 1998:44:103-8.

21. Xu XJ, Ferguson MR, Popov VL, Houston CW, Peterson JW, Chopra AK. Role of a cytotoxic enterotoxin in Aeromonas-mediated infections: development of transposon and isogenic mutants. Infect Immun. 1998;66:3501-9.

22. Suarez G, Sierra JC, Sha J, Wang S, Erova TE, Fadl AA, Foltz SM, Horneman AJ, Chopra AK. Molecular characterization of a functional type VI secretion 
system from a clinical isolate of Aeromonas hydrophila. Microb Pathog. 2008; 44:344-61.

23. Grim CJ, Kozlova EV, Ponnusamy D, Fitts EC, Sha J, Kirtley ML, van Lier CJ, Tiner BL, Erova TE, Joseph SJ, et al. Functional genomic characterization of virulence factors from necrotizing fasciitis-causing strains of Aeromonas hydrophila. Appl Environ Microbiol. 2014;80:4162-83.

24. Albarral V, Sanglas A, Palau M, Minana-Galbis D, Fuste MC. Potential pathogenicity of Aeromonas hydrophila complex strains isolated from clinical, food, and environmental sources. Can J Microbiol. 2016;62:296-306.

25. Libisch B, Giske CG, Kovacs B, Toth TG, Fuzi M. Identification of the first VIM metallo-beta-lactamase-producing multiresistant Aeromonas hydrophila strain. J Clin Microbiol. 2008;46:1878-80.

26. Fosse T, Giraud-Morin C, Madinier I. Phenotypes of beta-lactam resistance in the genus Aeromonas. Pathol Biol (Paris). 2003;51:290-6.

27. Zhiyong Z, Ziaoju L, Yanyu G. Aeromonas hydrophila infection: clinical aspects and therapeutic options. Rev Med Microbiol. 2002;13:151-62.

28. Lamy B, Kodjo A, colBVH Study Group, Laurent F. Prospective nationwide study of Aeromonas infections in France. J Clin Microbiol. 2009;47:1234-7.

29. Bhaskar M, Dinoop KP, Mandal J. Characterization of ceftriaxone-resistant Aeromonas spp. isolates from stool samples of both children and adults in Southern India. J Health Popul Nutr. 2015;33:26.

30. Neuwirth C, Siebor E, Robin F, Bonnet R. First occurrence of an IMP metallobeta-lactamase in Aeromonas caviae: IMP-19 in an isolate from France. Antimicrob Agents Chemother. 2007;51:4486-8.

Ready to submit your research? Choose BMC and benefit from:

- fast, convenient online submission

- thorough peer review by experienced researchers in your field

- rapid publication on acceptance

- support for research data, including large and complex data types

- gold Open Access which fosters wider collaboration and increased citations

- maximum visibility for your research: over $100 \mathrm{M}$ website views per year

At $\mathrm{BMC}$, research is always in progress.

Learn more biomedcentral.com/submissions 